\title{
An Engineering Approach for Project Scoping
}

\author{
Ofer Zwikael, John Smyrk \\ School of Management, Marketing and International Business \\ ANU College of Business and Economics \\ The Australian National University \\ Canberra, ACT, Australia \\ (Ofer.zwikael@anu.edu.au)
}

\begin{abstract}
Benefit realization is becoming a critical performance criterion measure for project success. However, the mechanism through which outputs eventually become outcomes has not yet been identified. This paper proposes that an output utilization mechanism is needed to explain the cause and effect relationship between a project's outputs and target outcomes. The paper describes such a utilization mechanism, and discusses its role in the generation of target outcomes. By expanding on the concept of output utilization, the paper suggests the utilization map as a new tool for project scoping.
\end{abstract}

Keywords - Project Scoping, target outcome, output utilization, empirical study, synthetic outcome

\section{INTRODUCTION}

Projects' outcomes are influenced by the delivery of its outputs - defined as the artifacts that are produced from the work of the project (Zwikael and Smyrk, 2011) - although the suggested forms of influence vary widely. Some empirical studies reveal a positive correlation (suggesting a casual relationship) between efficient delivery of outputs and customer satisfaction with project outcomes (Dvir and Lechler, 2004; Lipovetsky et al., 1997; Pinto, 1986). Dvir et al. (2003) found that fulfilling the functional requirements and meeting technical specifications of outputs is related to the realization of project benefits. Dvir and Lechler (2004) also found that customer satisfaction was related to completion of projects on time and within budget.

\section{PROJECT SCOPING}

Project scoping is a critical project process (Kankonen, 1999; Juan and Lidon, 2011; Zwikael and Globerson, 2006), which determines the outputs to be produced during the project. This process is undertaken early in a project initiation (Juan and Lidon, 2011) and provides the foundation of the business case. Because scope establishes a project's boundaries, it directly influences outcomes and outputs, as well as duration, cost and level of risk (Zwikael and Smyrk, 2011). If stated appropriately, a project's scope enables one to decide, in effect, "where the boundaries of the project lie".

Because the resources required by a project are implied by the work that must be undertaken and because that work is implied by the outputs that are to be delivered, we can conclude that a list of committed outputs provides an unambiguous declaration of project scope. Thus a project is scoped if and only if its outputs are defined.
Two conditions must be met if a project's outputs are to be defined: they are formally identified (as a list), and, for each, certain required characteristics (that we call its "fitness for purpose" features), are also set (Zwikael and Smyrk, 2011). At this point we foreshadow a later discussion of a related issue "How do we know if the declared scope of a project is 'correct'?".

Early in their conceptualization, all projects face the scoping problem (Zwikael and Smyrk, 2011): "Of all the lists of outputs (each with a supporting list of fitness-forpurpose features) that might be assembled for a project which one represents the most appropriate scope of the proposed initiative?".

The literature is generally silent on this problem. The Guide to the Project Management Body of Knowledge (PMI, 2008), suggests four tools and techniques to be used for project scoping: expert judgment, product analysis, alternatives identification, and facilitated workshops. PRINCE2 (OGC, 2007) provides scoping framework which can be applied to any type of project. This involves: (1) Establishing what products are needed, (2) Determining the sequence in which each product should be produced, (3) Defining the form and content of each product, and (4) Resolving what activities are necessary for their creation and delivery. However, these processes provide no guidance on how the correctness of scope should be tested.

The literature shows that project success is sensitive to scope (Kankonen, 1999; Juan and Lidon, 2011). Because project success is measured by the extent outcome are realized (Zwikael and Smyrk, 2011), it becomes critical to set the scope for a project that maximizes outcomes realized from a project. In other words, if all possible scopes for a project can be conceptually ranked according to their correlation with achievement of target outcomes, then the scoping problem takes the form of setting a scope that is more highly correlated than others. If not solved satisfactorily, scoping problem will result in either of unsatisfactory situations: underscoping, whereby the current outputs cannot support target outcomes and overscoping, whereby the current scope includes redundant outputs (or outputs with superfluous features).

We propose two methods to solve the scoping problem - one for natural outcomes and the other for synthetic outcomes. In the case of projects with natural outcomes, scoping appears to be relatively simple. The existence of strong causality between outputs and target outcomes means that scoping the project collapses into

978-1-61284-449-7/11/\$26.00 C2011 IEEE 
one of identifying those outputs that "cause" the desired end effects using established techniques such as the Ishikawa (fishbone ) Diagram. By way of contrast, scoping projects with synthetic outcomes is far from trivial-requiring an analytical model of the weak causal mechanism that relates outputs to outcomes-a mechanism that we call "utilization".

Consequently, an additional research question arises "How can a better understanding of the relationship between outputs and natural outcomes be used to set and validate the scope of a proposed project?". The objectives of this paper are, therefore, to explore the relationship between a project's outputs and desired outcomes and use this knowledge to better define and scope new projects. To address this issue we discuss in turn: a causal model that explains how outcomes emerge from a project, a tool to set and validate the scope of a project in terms of its outputs and an experiment to assess the effectiveness of that tool.

\section{OUTPUT UTILIZATION}

The literature emphasizes the role of customers in business transactions for the creation of organizational value. For example, Lepak et al. (2007: 182) suggest that a target user is the focus of value creation. Makadok and Coff (2002) claim that value creation requires a theory of consumer utility.

Organizational value can be conjectured or realized (Pitelis, 2009). A development of a unique product or service in projects only creates conjectured value to the funding organization. The conjectured value will become realized only through utilization of project outputs. For example, a document that describes an improved service process (project output) has conjectured value to the organization, which will be realized when salesperson and customers will start utilizing the new process.

\section{A.The utilization mechanism}

We propose that synthetic outcomes are generated through the utilization of outputs by particular stakeholders. The Sydney Cross City Tunnel is a case in point. The declared target outcome was "reduced congestion" (on the surface streets of the Sydney CBD). Two prominent outputs from this initiative were the tunnel itself and a business unit (to operate the facility when it became operational). Generation of the target outcome is clearly sensitive to the extent that motorists elect not to utilize the tunnel (and continue crossing the city using surface streets instead). We define a project customer as an entity who generates a target outcome as a by-product of utilizing a project output (often in some operational setting). This definition separates a project customer from a beneficiary or funder (although, clearly, in certain circumstances, one entity could qualify simultaneously as all three). Natural outcomes, on the other hand, arise regardless of any utilization of outputs by customers.

\section{B.The mediating effect of utilization}

There are various factors that can influence the extent to which utilization occurs. The first is related to the freedom of the customer not to utilize - or, equivalently, to the severity of the constraints that surround that freedom. If, for example, all surface streets of the Sydney CBD remain available to motorists after the opening of the tunnel, then they are free not to utilize it. The effects of utilization constraints on the customer's freedom not to utilize will range between two extremes: at one end the effect is zero-while at the other it is total. The second concerns the fitness-for-purpose of the project's outputs. If the fire control system was shown to be unreliable, then motorists would be less inclined to utilize the tunnel. The "quality" effect can be effectively addressed by incorporating an appropriate quality management plan into the project. The third concerns the predisposition of the customer to utilize the outputs. So if Sydney motorists became antipathetic towards the tunnel (which in fact happened), then, regardless of the quality of the outputs, motorists will avoid utilization. The "customer predisposition" effect can be partly addressed through a stakeholder engagement plan. Finally there is "bad luck". For example mine subsidence forces extended closure of one lane for an extended period of time. Such factors could be partly mitigated with a risk management plan. Despite the application of quality plans, engagement strategies and risk management, there is still no guarantee that target outcomes will be achieved.

\section{A UTILIZATION-BASED PROJECT SCOPING PROCESS}

We propose a scoping technique based on an analytical model of utilization involving the following sequence of steps: (1) Assemble an initial (tentative) statement of scope, (2) Refine/validate the statement of scope using a utilization map, and (3) Define target outcomes and outputs.

A.The statement of scope

A statement of scope serves as the foundation of a business case. It has three components: an objective statement, a list of target outcomes and a list of necessary outputs. The objective statement which provides a succinct answer to the question "Why is this project to be funded?". For example, a valid objective statement for Sydney's Cross City Tunnel would be "The objective of this project is to reduce congestion in the CBD". Supporting the objective statement is a list of target outcomes. We propose four criteria for a desirable outcome that make it suitable for targeting: it should be important (to the funder), it must be measurable (even if only qualitatively), there must be a plausible link with the projects outputs (further confirmed in the utilization map) and the lag between production of outputs and the generation of target outcomes must be acceptable to the funder. A valid target outcome for the Sydney Tunnel would be "Reduced numbers of vehicle travelling across the city through the CBD". The list of outputs for that same project included: the tunnel (with its supporting infrastructure-such as the traffic control system), a commercial business entity (to operate the tunnel) and a 
suite of operational processes (such as an emergency evacuation procedure).

At this point it is important to explain why target outcome usually begin with the participial adjectives "increased" or "decreased". In the case of the Sydney Tunnel, there are three scenarios that are relevant to a business case supporting the proposal for funding: (1) the "now" scenario (framed in terms of the current daily count of vehicles travelling across the City through the CBD), (2) the "yes" scenario (framed in terms of the daily count of vehicles travelling across the City through the CBD if the tunnel is built and operating successfully) and (3) the "no" scenario (framed in terms of the daily count of vehicles travelling across the City through the CBD if the tunnel is not built). Target outcomes are defined in terms of the difference between the "no" and "yes" scenarios, not as the difference between the "now" and "yes" scenarios. In other words target outcomes represent changes between two future scenarios rather than timebased changes.

\section{B.The utilization map}

The utilization map is a device (based on a model of utilization) that is intended to validate project scope, by making sure that the scoping statement meets two criteria: that all outputs required by utilization for the generation of target outcomes have been included and that none of the identified outputs are redundant. It does this by identifying the project customers who generate particular target outcomes through their utilization of specific outputs. The utilization map is presented as a table in which: (1) each column is associated with a target outcome, (2) each row is associated with an output and (3) each cell shows which customer/customers (if any) utilize the output on the left to generate the outcome at the top.

Zwikael and Smyrk (2011) suggest that cells in the utilization map can finish up with any combination of three sorts of entry:

- Blank (the cell is empty). No one utilizes the output at the left to generate the outcome at the top. For example in Table 8, no one utilizes the panel of preferred suppliers to reduce payment times to suppliers.

- A single utilization. One customer utilizes the output to realize an outcome. For example, only procurement staff utilize the programs of professional development to reduce costs of procurement. (Although there are many staff, collectively they make up a single customer entity)

- Multiple utilizations. Several customers utilize the output to realize an outcome. For example, reduced payment times to our suppliers require that the new procurement processes be utilized by both the procurement staff and suppliers.

Validating a project's scope is done by examining the utilization map in the following way:

For each outcome column:

Check to see if the combined contributions of all utilizations listed anywhere under this outcome represent an acceptable level of outcome achievement. The extreme case is where a column of the utilization map is empty (that is no customer is identified as utilizing any output to generate this outcome). This could indicate missing outputs (that is, the project is currently underscoped).

For each outputs row:

Check if the combined contributions of all utilizations listed anywhere against this output represent a significant role for it. The most extreme case is where the row of the utilization map is empty (that is no customer is identified as utilizing this output at all). This could indicate that the output is redundant (that is, the project is currently overscoped).

\section{REFERENCES}

[1]Dvir, D., Lechler, T. (2004). Plans are nothing, changing plans is everything: The impact of changes on project success. Research Policy, 33 (1), 1-15.

[2]Hanisch, B., Wald, A. (2011). A project management research framework integrating multiple theoretical perspectives and influencing factors. Project Management Journal, 42 (3), 422.

[3]Lechler, T., Dvir, D. (2010). An Alternative Taxonomy of Project Management Structures: Linking Project Management Structures and Project Success. IEEE Transactions on Engineering Management, 57 (2), 198-210.

[4]Lepak, D. P., Smith, K. G. and Taylor, M. S. (2007). Value creation and value capture: A multilevel perspective'. Academy of Management Review 32/1: 180-94.

[5]Lipovetsky, S., Tishler, A., Dvir, D., Shenhar, A., 1997. The relative importance of defense projects success dimensions. R\&D Management 27, 2.

[6]Makadok, R. and Coff, R. (2002). The theory of value and the value of theory: Breaking new ground versus reinventing the wheel'. Academy of Management Review 27/1: 10-13.

[7]Malach-Pines, A., Dvir, D., Sadeh, A. (2009). Project manager-project (PM-P) fit and project success. International Journal of Operations and Production Management, 29 (3), 268. [8]OGC - UK Office of Government Commerce. 2007. Managing successful programs. The Stationery Office, Norwich, UK.

[9]Pinto, J.K., 1986. Project implementation: a determination of its critical success factors, moderators and their relative importance across the project life cycle. Dissertation at the University of Pittsburgh, Pittsburgh.

[10]Pitelis, C. (2009). The co-evolution of organizational value capture, value creation and sustainable advantage. Organization Studies, 30(10), 1115-1139.

[11]Shenhar, A. J., Dvir D. (2007). Reinventing Project Management: The Diamond Approach to Successful Growth and Innovation. Harvard Business School Press.

[12]Shenhar, A., Dvir, D. (1996). Toward a typological theory of project management. Research Policy, 25, 607-632.

[13]Zwikael, O., A. Sadeh. (2007). Planning effort as an effective risk management tool. Journal of Operations Management, 25 (4), 755-767.

[14]Zwikael, O., Globerson, S. (2004). Evaluating the quality of project planning: a model and field results. International Journal of Production Research, 42 (8), 1545-1556.

[15]Zwikael, O., Globerson, S. (2006). From critical success factors to critical success processes. International Journal of Production Research, 44 (17), 3433 - 3449.

[16]Zwikael, O., Smyrk, J. R. (2011). Project Management for The Creation of Organisational Value. Springer-Verlag, London, UK. 